\title{
Embodied Learning in Immersive Smart Spaces
}

\author{
Mirko Gelsomini \\ Politecnico di Milano \\ Milan, Italy \\ mirko.gelsomini@polimi.it
}

\author{
Giulia Leonardi \\ Politecnico di Milano \\ Milan, Italy \\ giulia.leonardi@mail.polimi.it
}

\author{
Franca Garzotto \\ Politecnico di Milano \\ Milan, Italy \\ franca.garzotto@polimi.it
}

\begin{abstract}
This paper presents the design and evaluation of IMAGINE, a novel interactive immersive smart space for embodied learning. In IMAGINE children use full-body movements and gestures to interact with multimedia educational contents projected on the wall and on the floor, while synchronized light effects enhance immersivity. A controlled study performed at a primary school with 48 children aged 6-8 highlights the educational potential of an immersive embodied solution, also compared to traditional teaching methods, and draws some implications for smart-space technology adoption in educational contexts.
\end{abstract}

\section{Author Keywords}

Children; Education; Embodied Learning; Kinect; Immersive Environment; Smart Spaces; Primary School

\section{CCS Concepts}

-Human-centered computing $\rightarrow$ Field studies; Virtual reality; Interactive systems and tools; Empirical studies in interaction design; -Social and professional topics $\rightarrow$ Children; Student assessment; •Applied computing $\rightarrow$ Interactive learning environments; Education; Collaborative learn;

\section{INTRODUCTION}

This work investigates the pedagogical potential of IMAGINE (Immersive Multimodal Ambient Gymnasium IN Education), an immersive interactive environment for educational purposes where children are exposed to auditory and visual stimuli (multimedia projections and lights), activated by performing mid-air gestures and movements in a digitally enhanced physical space. The system is remotely controlled by the teacher, who can customize each experience according to group's or individual's needs, modifying intensity, duration and orchestration of stimuli and action-stimulus sequences.

Children's experiences in IMAGINE mimic some educational processes traditionally used in the classroom, exploiting the potential of immersive embodied interaction for learning. This approach is grounded on theories that recognize the relationship between physical activity and cognitive processes $[26,53,28,84]$.

Permission to make digital or hard copies of all or part of this work for personal or classroom use is granted without fee provided that copies are not made or distributed for profit or commercial advantage and that copies bear this notice and the full citation on the first page. Copyrights for components of this work owned by others than the author(s) must be honored. Abstracting with credit is permitted. To copy otherwise, or republish, to post on servers or to redistribute to lists, requires prior specific permission and/or a fee. Request permissions from permissions@ acm.org. CHI '20, April 25-30, 2020, Honolulu, HI, USA.

(C) 2020 Copyright is held by the owner/author(s). Publication rights licensed to ACM ACM ISBN 978-1-4503-6708-0/20/04 ...\$15.00.

DOI: https://doi.org/10.1145/3313831.3376667
According to the hypothesis that embodiment can enhance the learning process, we have designed and developed an educational didactic experience within IMAGINE aimed at facilitating learning of "factual knowledge" on a curricular subject. The experience is modeled following Kolb's experiential learning process, which is based on the cyclic sequence of "experience-reflection-learning" phases. According to Kolb, knowledge acquisition involves a process of problem solving and reaches the clarifying solution through the observation of the reality and elaboration of hypotheses.

The paper describes an empirical research devoted to compare the learning benefit of an immersive embodied approach against a traditional paper-based classroom approach. To address this issue, seventy first and second grade primary scholars were involved in a controlled study, in which two experimental conditions were considered: a traditional teaching method and an IMAGINE-based teaching experience.

The rest of the paper is organized as follows. In Section "Background and Related Work", we provide an overview of the theoretical approaches related to embodied interaction and on current solutions adopting embodied learning. We then present the design and development of IMAGINE. The following sections are devoted to the description of a controlled empirical study in which we evaluated IMAGINE potential in a primary school, and to the discussion of the main results. We conclude the paper proposing some design recommendations to enhance the educational potential of IMAGINE and, more generally, of smart spaces for learning, and outline future research directions.

\section{BACKGROUND AND RELATED WORK}

Embodied cognition theories emphasize the relationship between physical activity and cognitive processes and are supported by a growing body of evidence from psychology and neuro-biology $[90,91]$. These approaches embrace a perspective on learning as, at least partly, situated (or embedded) process, where the interaction of the body in a real spatial context is a major gateway to cognition [26]. These notions take roots from Piaget [68], who described a child acquiring "the practical knowledge which constitutes the substructure of later representational knowledge" particularly during the first sensorimotor developmental stage. This interpretation changed 30 years later leading to the now common proposition that "sensorimotor activity is not merely a stage of development that fades away in more advanced stages, but rather is thoroughly present in thinking and conceptualizing" $[66,70]$. According to recent neuro-biological studies, our brain acquires notions and relationships faster if these elements are lived physically and emotionally in first person $[51,53]$. 
An additional theoretical underpinning of our work is the approach proposed by the American educator David Kolb, who defined the steps that make learning effective and efficient. His method is based on the assumption that learning is circular [52, 54], and his learning cycle, which defines the phases of experiential inductive learning, offers a model for educational practice. The cycle is composed by 4 sequential phases: concrete experience, reflective observation, abstract conceptualization and active experimentation. During the concrete experience phase, participants explore knowledge through simulations, whereas, during reflective observation, they observe, reflect and interpret what emerged during the experience through discussion and brainstorming. The reflection is followed by abstract conceptualization, which aims to produce and outline concepts by extending them through models and lessons. Finally, during the active experimentation, the knowledge and skills acquired in new situations are verified through simulations, games and challenges. This last phase translates into a new concrete experience and the knowledge acquired produces new ways of doing and thinking, so that the cycle can start again.

Educational research incorporating students' bodily experiences, often referred to as embodied learning, is receiving considerable attention in the research community [57, 62]. Embodied learning was initially achieved via controllers, mouse devices, or joysticks $[88,80]$ and more recently through the use of motion-based sensors such as Microsoft Kinect, Nintendo Wii, Intel RealSense. These devices can interpret the learner's body movements and gestures (e.g., clicking, grasping, pointing, walking, or balancing) to interact with the multimedia contents $[6,43,56]$. Some very recent implementations of embodied learning solutions see the use of entire environments orchestrating large screens and motion-based technologies, in order to achieve a greater learning effectiveness $[12,1,47,31,30,3]$.

Within this domain, a large number of studies have been performed incorporating embodied activities (in some cases involving minimal movements $[4,15,25,33,42])$ in various field, such as mathematics $[81,72,59,19]$, physics $[9,37,7$, $36,45,60,61]$, chemistry [46], science [2, 16], vocabulary and language acquisition [57,69], reading comprehension $[35,34]$, sound concepts [10, 67, 39, 41]. These studies aim at evaluating the effectiveness of embodied education and partially confirm the theory on the benefits of learning by doing and the role of active experience in grounding concepts [63, 46, 72].

Most of these studies evaluate only the temporary learning impact and rarely assess their actual benefit on students in comparison with a control group. Some other studies do not completely support the thesis that higher levels of body engagement lead to better learning outcomes than those featuring lower bodily involvement (e.g. observing, using paper and pen, or performing minimal gestures) [81, 75, 5, 64]. Particularly, Johnson-Glenberg et al.[48] could not find a significant learning advantage for higher embodiment levels in the short term, yet they were able to detect higher retention with a 1 week-delayed test for those participants who experienced an higher level of embodiment.

Overall, existing research on embodied learning technologies has been disparate, driven largely by specific technical innovations and constraints, and supported by limited statistical evidence about how embodied and immersive solutions, such as full-body interaction in smart environments, affects learning [21, 37, 55, 62]. Indeed, to our knowledge, no studies have explored the effects that educational experiences in immersive embodied environments might have on long term memory skills.

The empirical study performed in our research focuses on comparing children's information retention (defined as the process of "keeping memory in human memory stores" [77]) when learning in two teaching environments: an immersive context enhanced with embodied interaction (IMAGINE) and a traditional classroom one. We explored both "short term retention" - the retention of information lasting a few days (proportional to short-term memory) - and "long term retention" - the one lasting for more than 1 month (relating to long-term memory). In particular, we studied long-lasting retention of information in the form of semantic memory [50] that, with episodic memory, forms the declarative/explicit memory [79]. Declarative memory is essential for learning new information, as it functions as a site for storage and retrieval of learned knowledge [82].

\section{Taxonomies}

Various taxonomies have been defined that identify various design dimensions and features to characterize technology for embodied learning (Table 1). In this section, we outline the most relevant ones reported in the existing literature and pinpoint with a * those items that do not apply to IMAGINE.

Malinverni et al.[63] focus their taxonomy on the factors of physical configuration, namely technologies involved and their characteristics, number of users, input data, i.e. nature of the interaction required by the system and mapping, degree to which user actions are related with learning.

Johnson-Glenberg et al.[46] identify in motoric engagement, gestural congruency and perception of immersion the features of their classification. Full body motoric engagement is achieved through locomotion, so a technology that affords the learner the opportunity to ambulate will score highest (lower if it allows the use of partial body); gestural relevancy is determined by the level of relevance of the gesture as it maps to the content to be learned, as Malinverni's mapping; immersion level ranges from a minimum brought by tablets and computers to a maximum reached by large screens.

Melcer et al.[65] presents a framework with 7 design dimensions. Physicality describes how learning is physically embodied, transforms conceptualize a space and the relationships between physical/digital actions and physical/digital effects, mapping addresses how input is spatially mapped to output, correspondence* refers to the degree to which the physical properties of objects are closely mapped to the learning concepts, mode of play specifies how individuals socially interact, coordination highlights how individuals socially coordinate their actions, environment refers to the characteristics of the environment (physical, mixed, virtual).

Duijzer et al.[26] address 8 mediator categories, namely real-world context indicating to experiences of students with or in the real world, multimodality referring to interaction 
modalities, linking motion to concept as in JohnsonGlenberg's gestural congruency, multiple representations* conceptualizing the multiple representations of motions, semiotics $*$ describing the use of meaning-supported sign systems, student control addressing how students are in control of the learning environment, bodily involvement giving indication of students' engagement with a movement (ranging from an action of the whole body to observing the movement of others), immediacy highlighting the temporal sync of motion with the content, attention capturing* characterizing the aspects that capture students' attention, cognitive conflict* specifying to conflicting conceptions.

Bowman et al.[14] focus on virtual reality solutions and on their technical components such as field of view (size of visual field), field of regard (total size of the visual field surrounding the user), display size and resolution, stereoscopy*, head-base rendering*, realism of lighting, frame rate* and refresh rate*.

Table 1 summarizes the main design dimensions of the cited taxonomies and highlights (in green) how they match the design solutions of IMAGINE.

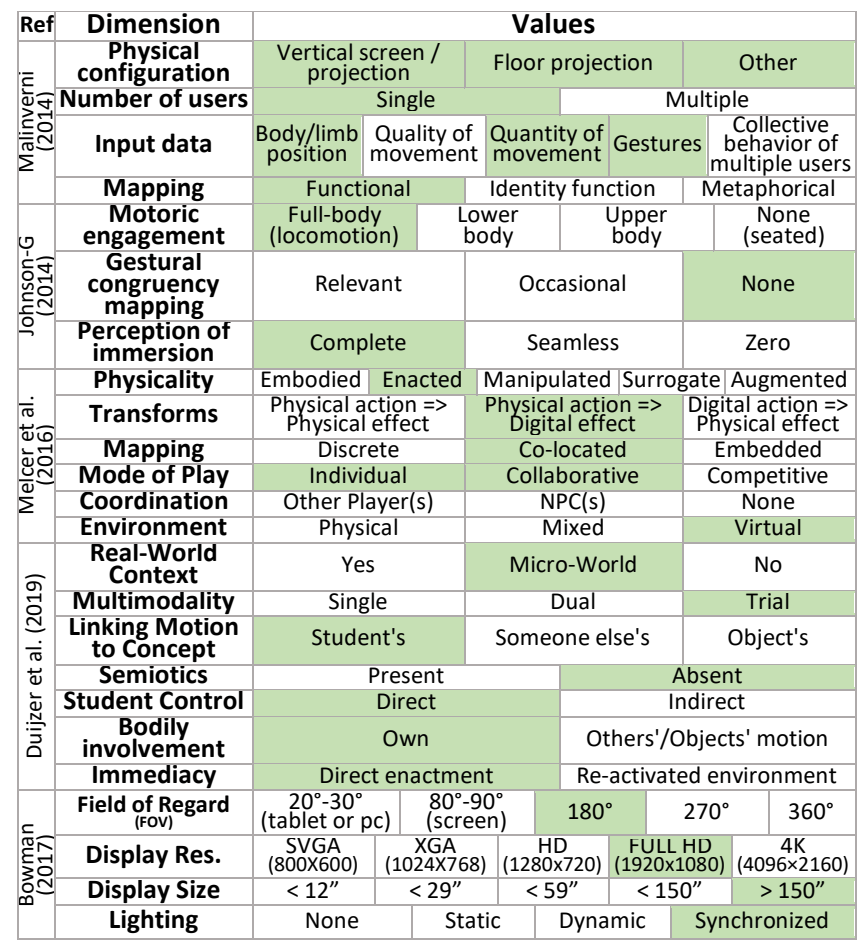

Table 1: Main design taxonomies for embodied learning; dimensions that apply to IMAGINE are in green

\section{IMAGINE}

In the "Immersive Multimodal Ambient Gymnasium IN Education" (IMAGINE), children's experience is motion-based and immersive, involves different types of interaction and includes tasks and rewards - remotely controlled by the teacher through a tablet - based on different visual and auditory stimuli. IMAGINE makes use of a vertical full-hd projection, a full-hd floor projection, and synchronized colored lights (i.e. they change according to the shown content) with a field of regard of 180 degrees (vertical) [14]. The environment can be considered as a micro world, a virtual representation of a real but less complex world $[26,76]$ with a size of about 30 sqm.

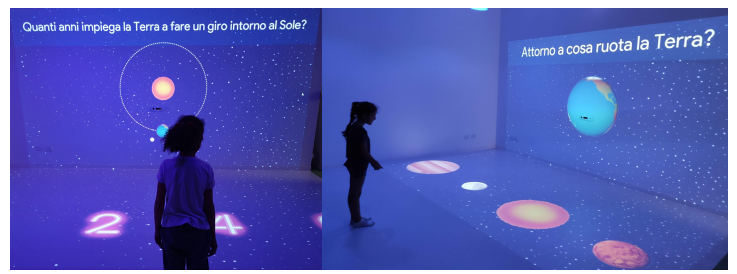

Figure 1: The IMAGINE environment

Microsoft Kinect v2 was chosen as the motion sensor given its inexpensive and compact hardware (hidden inside the drywall), its online community, its ease of code maintenance between device versions, and its high precision in recognizing up to six player's movements and gestures in the space. Current IMAGINE activities were designed so that only one student could interact with the educational contents [63], but many students can collaborate in the educational experience while waiting for their turn [65]. Each of the 5 interaction types developed considers a different input (player's position and motion, hand movement, gestures such as drag \& drop, selection), perceived by the system in terms of quantity of movements, leaving aside qualitative measurements such as gestures speed or flow [63]. The mapping between player's motion and educational contents, shown on one of the projections, is functional [63] and gesturally incongruent [46], a concept to different taxonomies that indicates that user actions and mapping do not relate semantically with the content but only have a functional meaning. The interaction is contiguous and co-located (i.e., input and output are close and an action triggers an output that is directly adjacent or overlaid [65]), directly enacted (i.e., the student's physical action is directly linked to the digital content and does not depend to any other mediating factor such as other players or objects $[26,65])$, immediate (i.e., referring to a cognitive activity where perceptual-motor interaction in the presence of direct environmental stimuli is instantaneous [26]), and semiotics is absent (i.e., "gestures in the environment are not explicitly used to signify meaning. [...] Content and the result of the interaction is meaning" [26]).

According to the identified taxonomies, IMAGINE can be classified as having: enacted physicality (i.e., action knowledge through physical action) [65]; high bodily involvement comprising the alternation of motor execution (i.e., direct full body experience) and motor mirroring (i.e., the mirror-neuron system is activated when observing movements made by others) [26]; trial multimodality (at least two of the modalities of seeing, hearing, touching, imagining, or motor actions are simultaneously activated [26]) and a medium level of immersivity (offering a field of regard of $180^{\circ}$ with two full-hd projections of over 150 inches and synchronized lights [14, 46]). Given Johnson-Glenberg et al.'s definition of "embodied learning" as the result of the combination of motoric engagement, gestural congruency and perception of immersion[46], and considering the classification of IMAGINE as providing full-body motoric engagement (i.e., "students move their bodies from one point to another and exert control over the graphical representation of the movement"[26]), low gestural congruency, and medium level of immersivity, we would characterize our system as a " medium to low embodiment" learning environment. 


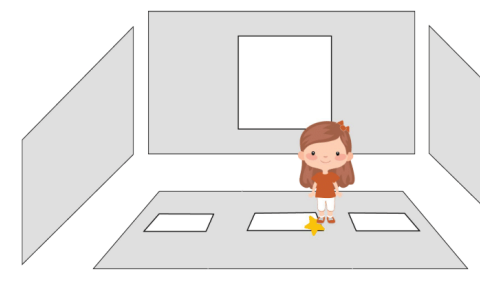

a) Selection

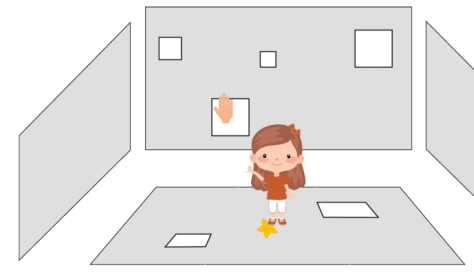

d) Identification (Color and Shape)

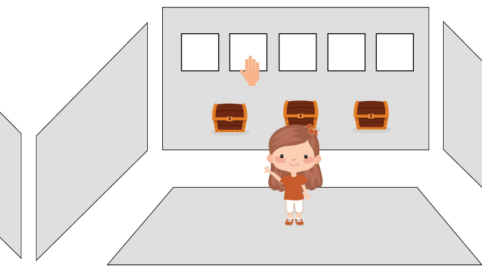

b) Classification

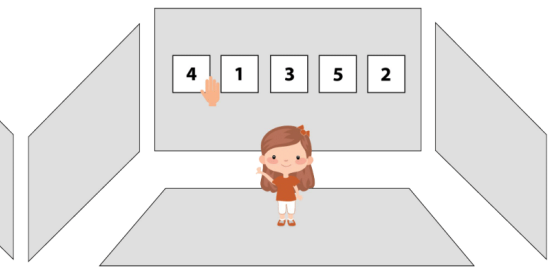

c) Reordering

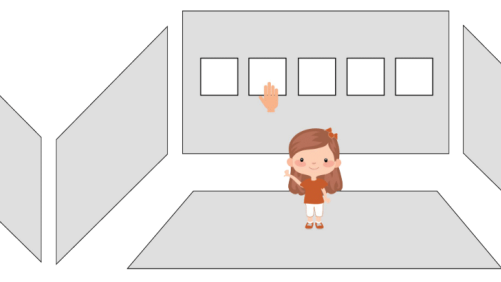

e) Identification (Position)

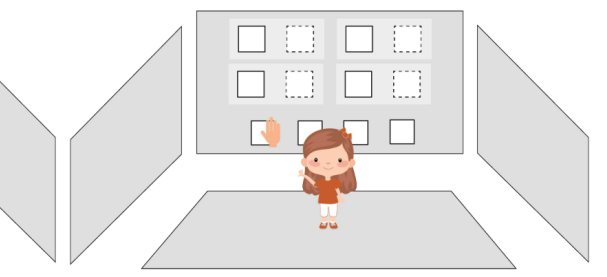

f) Association

Figure 2: Interaction Types

\section{Educational Experience}

IMAGINE's learning experience is inspired by Kolb's learning cycle $[52,54]$ and divided into four different phases, following the inductive process of the STAR Legacy module [44]: Challenge, Initial Thoughts, Resources and Assessment. Children are welcomed in IMAGINE and the teacher presents the topic that will be discussed during the lesson making use of images projected on the wall and on the floor (Challenge phase). She then collects students' questions and elicits children's discussion from previous knowledge (Initial Thoughts phase). During the Resources phase, a 5-minutes video is projected to the wall: when the projection ends, children are called in turns, one at a time, to "perform a task", which consists of answering a proposed question using one of the given interaction types presented in Section 3.2. A task is completed only when the child chooses the right answer. Lastly, children are evaluated on their acquired knowledge (Assessment).

\section{Interaction types}

The structure of each single task was designed on the basis of the textbooks currently used in primary schools. In this regard, we have collected primary school's books and extracted and analyzed the different types of exercises. Five main types answer modalities emerged, frequently repeated in the exercise section of the different topics, although aimed at different educational objectives and including different graphic contents. Therefore, five main interaction types are defined for IMAGINE:

a) Selection. The selection pattern presents the user with an explanatory image of the question on the front projection and a variable number of response option cards on the floor projection (Figure 2a). A star projected on the floor, placed immediately in front of the user's feet, follows the student's movements. In order to select the chosen answer, the child must place the star, and consequently herself, over the card corresponding to the option deemed correct. The child confirms her decision standing over the card for at least 3 seconds. A loading bar appears to provide a feedback on the performed action. b) Classification. The classification pattern presents the user with a set of images and a series of labelled boxes, both projected on the front projection. A virtual superimposed hand appears, following the preferred user's hand (Figure 2b). The user is asked to grab (close hand for selection), drag (move hand) each of the images presented at the top of the screen and release (open hand) each of them in the correct category, shown in the form of a box at the bottom of the frontal screen. c) Reordering. The reordering pattern presents the user with a series of images randomly ordered on the front screen. A virtual superimposed hand appears, following the preferred user's hand (Figure 2c). The user is asked to reorder the option cards according to a sequence contextualized by the question. To reorder the cards, the child is required to grab, drag and release each element until the desired sequence is obtained. A pop-up will ask the user to confirm or cancel the response given after 5 seconds from her last interaction.

$d$-e) Identification. The identification pattern requires users to recognize a given object based on its characteristics. Two different types of identification are supported: Based on Color and Shape. A variable number of images are randomly distributed on both projections. The user has to identify the item requested in the question and select it by positioning her body (item positioned on the floor) or by moving and closing her hand (item positioned on the front wall) as shown in Figure $2 \mathrm{~d}$. Based on Position. A variable number of images are arranged neatly on the front screen. The user has to identify the item requested by the question by moving and closing her hand on its top as shown in Figure 2e.

f) Association. The association pattern presents users with four frames at the top and four items at the bottom of the front projection (Figure 2f). Each frame contains two slots, one housing an image and the other empty. The user is asked to associate (grab, drag and release) each bottom item with the images presented in the frames, placing the objects in the free frame slots. Once all objects are placed in the corresponding slots, a pop-up asks to confirm or retry. 


\section{EXPERIMENTAL STUDY}

In order to investigate whether IMAGINE can actually facilitate children's learning, a controlled experimental study was conducted. The study is based on the comparison between the learning process that takes place in a traditional classroom and the one occurring in IMAGINE, with the aim of comparing the impact on children's learning achievements. This study investigates a main research question:

Can learning benefit from an immersive embodied approach compared to a traditional paper-based classroom approach?

The underlying hypothesis for the research question is that differences in students' achievement might emerge when they receive two different instructional treatments. The participant children were pair-wise assigned according to performance on a pretest to a control group, subjected to the traditional practice based on the frontal book-based approach within the classroom, and an experimental group, subjected to the practice within IMAGINE environment.

Students were tested at the beginning of the study $\left(\mathrm{T}_{0}\right)$, after 1 day from the last learning session $\left(\mathrm{T}_{1}\right)$ and 50 days after it $\left(T_{2}\right)$ using the same Test Sheet described in the "Materials" section. Before the study, we defined those research variables able to answer the above question in terms of short and long term retention as described in the "Data Gathering" section.

\section{Participants}

Seventy primary school children (6-8 y/o, avg: $6.94,35$ females) of the Via Sturzo school in Cornaredo (Milan, Italy) were recruited for the study. The motivation behind the rationale regarding the age group lies in the simplicity of the interaction modes involved in the study. In order to identify the right target, we have previously presented the activities designated for the experimentation to children aged between 6 and 11 years old, observing how the simplicity of movements was perceived as limiting by older children, but appeared to be suitable for the first and second graders motor skills. A primary school teacher, unknown to all children, was recruited to lead lessons both in class and within IMAGINE, guiding and stimulating children to learn with the same verve.

\section{Materials}

\section{Exercises}

In order to allow children to practice the knowledge initially gained from viewing the explanatory video, we have created 62 exercises. Both IMAGINE and classroom activities used the same exercises, proposed in the same sequence and with the same graphical contents.

\section{Learning topic}

The instructional topic used for this study was identified in collaboration with teachers and pedagogists according to the following requirements.

- Novelty. The choice of the content had to be completely new to the students involved, thus avoiding potential threats to internal validity.

- Complexity. The choice of the topic was evaluated among those usually planned to be treated in a short period of time.
The difficulty of the topic was assessed on the basis of educational plans currently suggested by the Ministry of Education and by the School Curricula, ensuring a proposal that fell within participants' abilities.

- Measurability. In order to be able to identify the learning progresses, we tested and quantified children's knowledge about the topic before and after the training. Evaluating each child's starting level could reveal itself to be rather simple for a notional topic (the child knows or doesn't), definitely more complex for an oral comprehension or logic exercises, for which the individual previous training and inborn attitudes would play a key role in the resulting performances.

- Potential Exploitability. Among IMAGINE features, we can identify immersivity as the only characteristic whose employment and effectiveness is largely influenced by the proposed topic. We believe that subjects dealing with visual and realworld related topics - such as Geography and Science - can highly benefit from an immersive environment.

With these premises, we have oriented our choice towards the Solar System as a purely notional topic which contextualization we believe could extensively exploit children's abilities and IMAGINE's potential benefits. We first identified an appropriate video for the explanation phase of the chosen contents. In this regard, we chose a video from the European Space Agency (ESA), specifically created for teaching purposes. We then extracted the topics treated in it and generated exercises focused on these arguments.

\section{Test Sheet}

A paper-based test was designed, identical for all participants, with the aim of checking students' knowledge before and after the practicing sessions. The test consists of 12 questions of different complexity and different type (multiple choices, fill gaps, associations), each of which covering one or more educational objectives. The test was developed following the examples of tests currently assigned to primary pupils during the school year and was validated by teachers. One point was assigned to each correct answer, 0 to each wrong one, for a maximum achievable score of 45 . Grammatical mistakes and typos were not considered as errors.

\section{Procedure}

The study was planned to be carried out in two months, including lessons, training, and evaluations. We decided to base our analysis on school tests results, given their quantitative nature that enabled us to make comparisons between groups, across time, and with current national performance scores.

A Baseline Test $T_{0}$ was administered at the beginning of the first week, in order to establish homogeneous control and experimental groups.

Two learning sessions of 1 hour each were held in two separate days of the first week (same amount of time dedicated to similar topics at school).

A Short-Term Test $T_{1}$ and a Long-Term Test $T_{2}$ were provided on the last weekday of the first week and after seven weeks respectively. The procedure is depicted in Figure 3. 


\section{$T_{0}$ : Baseline Test}

Baseline test $\mathrm{T}_{0}$ was administered to all 70 participants. Children were positioned at reasonable distance and 4 supervisors were present with the aim of maintaining order and discouraging mutual suggestions among children. Questions were spoken aloud and children were given 30 minutes to complete the 12 questions, for an average of 2.5 minutes per task. No rush was given to students to finish in time. After the test, children were divided homogeneously with respect to the discriminating factors of score achieved in $\mathrm{T}_{0}$ and age into two groups of 35 participants each:

- Control group: Participants learn in classroom by practicing with paper-based exercises,

- Experimental group: Participants learn in IMAGINE by practicing with multimodal exercises.

For logistical reasons and given the limited capacity of both IMAGINE and the classroom, control and experimental groups were divided into 2 subgroups, homogeneous in terms of performances achieved in $\mathrm{T}_{0}$ and age, each of which consisted of 18 and 17 children (the size of a primary school class). For each of the aforementioned subgroups, two learning sessions were conducted.

\section{First Learning Session}

The first session was scheduled for the day immediately following $\mathrm{T}_{0}$. The two experimental subgroups entered IMAGINE for the first time, an hour each, while the two control subgroups attended their first session in classroom, an hour each. Thirty-one tasks were assigned to children, considering 1.5 minutes as the average execution time for each task, including the time taken by the child, within IMAGINE, to stand up and reach the game position; the interaction time, the time needed for any guide or additional explanation by the teacher; and the time required to identify the right answer. In parallel, the same time per task was considered reasonable for the oral management of the assignment within the classroom, including the reading of the question, the response time of the child and the time of verification by the teacher of the occurred collective understanding of information.

\section{Second Learning Session}

The second session was scheduled for the day after the first session with the same modalities, replacing the explanatory video with a 5 minute recap session. During the session, the remaining 31 tasks from the 62 generated as exercises were administered to students.

\section{$T_{1}$ : Short-Term Test}

The test was repeated identical to all 70 participants the day after the second session, in the same form and manner in which it was performed in $\mathrm{T}_{0}$.

\section{$T_{2}$ : Long-Term Test}

The test was repeated identical to all 70 participants 50 days after the Short-Term Test, in the same form and manner in which it was performed in $\mathrm{T}_{0}$ and $\mathrm{T}_{1}$. During the 50 days that elapsed between July $\left(T_{1}\right)$ and September $\left(T_{2}\right)$, due to the Italian summer holidays, study participants did not attend any lesson, neither were assigned homeworks related to the topic.

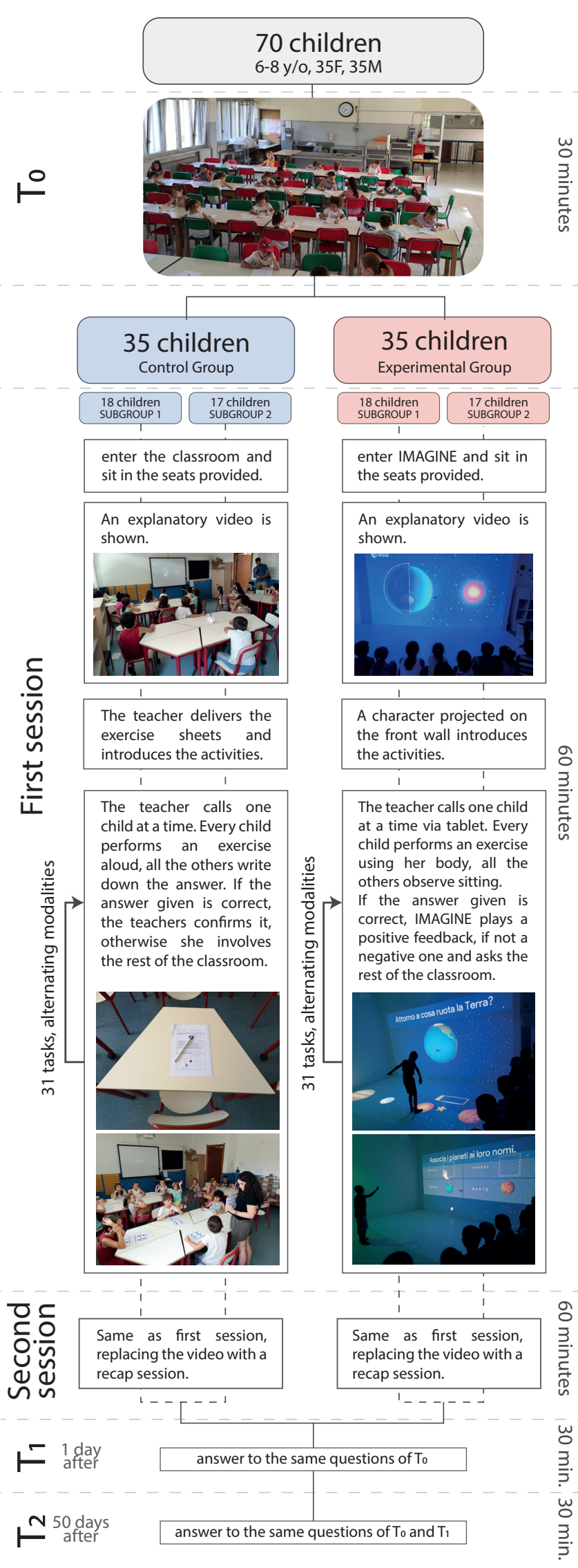

Figure 3: Controlled Study Procedure 


\section{DATA GATHERING AND ANALYSIS}

Test Scores were collected manually. Children's performances were not analyzed :

- Children scored above $60 \%$ in the baseline test $\left(T_{0}\right)$. This cutting threshold was defined according to the spread national educational practice for which this value is generally considered as "sufficiency", i.e. the limit above which school tests are deemed to have been passed by students.

- They were absent during on one or more training or testing sessions, which would hinder cause-effect relationship between studied contents and test scores.

\section{Research Variables}

For each group (control and experimental) we have measured the following research variables.

- Short term Retention (SR): average number of notions retained after 1 day $\left(\mathrm{T}_{1}-\mathrm{T}_{0}\right)$

- Long term Retention (LR): average number of notions retained after 50 days $\left(\mathrm{T}_{2}-\mathrm{T}_{0}\right)$

- Memory Loss (ML): average number of notions forgotten after 50 days $\left(\mathrm{T}_{2}-\mathrm{T}_{1}\right)$

- Content Retention Rate (CRR): average number of notions retained (LR) over Total Learning Time (TLT: 2 hours)

- Active Learning Turn Efficacy (ALTE): average number of notions retained (LR) over number of turns (T: 8). A turn corresponds to the time the child was called to actively participate to the learning session by answering in class or by performing the required action in IMAGINE

We assessed the significance of the difference of SR and LR between groups through a Two-way paired T-Test.

\section{Ethical considerations}

We carefully considered the ethical implications of the study. Confidentiality. A consent form, dealing with the use of data and images recorded during the study, was signed by both parents or legal tutor of each child involved. The information regarding participants were treated with the highest level of confidentiality, storing only the participant id and related tests results. Personal data were processed only to the extent that they were indispensable in relation to the objective of the study, in compliance with the provisions of current legislation on the protection of personal data and in accordance with the provisions of the general authorizations of the Guarantor Authority for the protection of personal data.

Psychological risks. In order to reduce the sense of frustration and discouragement, which could be caused by the difficulty and sometimes impossibility of correctly answering the proposed questions, due to the lack of specific knowledge, we presented children the test as an introductory process needed by the IMAGINE world to calibrate the activities for them. All children, comprised those in the control group, were invited to freely play in IMAGINE after the study period.

\section{RESULTS}

Figure 4 shows the baseline characteristics which led to the allocation of 35 children in the experimental group (in red) and 35 children in the control group (in blue). The average $\mathrm{T}_{0}$ score of children in the control group (CL) is 11.27, while the average $T_{0}$ score of children in the experimental group (IMG) is 11.18; the proportion of $1^{\text {st }}$ graders was slightly higher in both groups.
Among the sample, follow-up values were collected in 48 children, that is, $68,4 \%$ of the total. From the recruited 70 participants, two students (one per group) were excluded from the data analysis due to their high scores $(>60 \%)$. These students achieved an improvement $80 \%$ lower than the average scores (ceiling effect). One participant was excluded as she spontaneously informed the researchers to having discussed the treated topic with parents, deepening the knowledge gained from the exercise sessions, before taking the final test. Of the remaining 67 children, 19 were excluded due to their absence on days of either training or testing. This reduction in the sample led to analyse 26 children in the control group and 22 children in the experimental one.

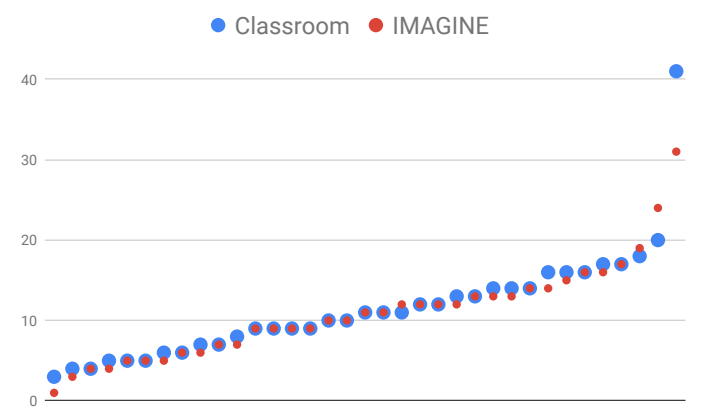

Figure 4: Control and Experimental Group Distribution

The immediate effect of the intervention after the two training sessions, which we refer as Short term Retention (SR), was evaluated based on changes in the values of the score obtained during $\mathrm{T}_{1}$ with respect to that achieved with the baseline test in $\mathrm{T}_{0}$, as depicted in Figure 5. SR was significant for both groups as they scored an average of 18 notions $\left(\mathrm{M}_{\mathrm{CL}}=\right.$ $\left.18.35, \mathrm{SD}_{\mathrm{CL}}=8.69, \mathrm{M}_{\mathrm{IMG}}=18.18, \mathrm{SD}_{\mathrm{IMG}}=6.52, \mathrm{p}<0.0001\right)$.

We categorized each of the two groups into tertiles based on SR scores. The first tertile can be traced back to the children who have retained fewer concepts and, therefore, probably those who have encountered more difficulties. The third tertile is associated to those children who acquired more concepts, which we can assume have had fewer difficulties. Consequently, the second tertile represents the group of children whose SR is in the middle range.

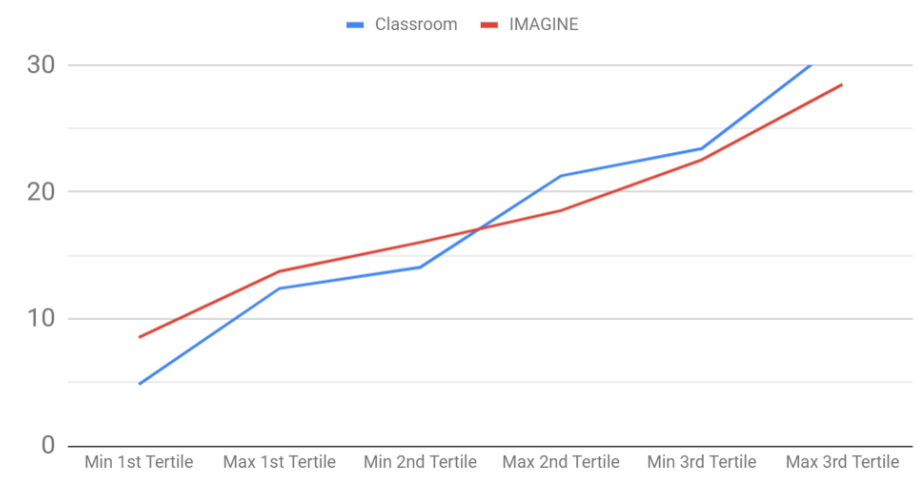

Figure 5: Classroom vs IMAGINE Short term Retention (SR) 
Children belonging to the first tertile retained an average of 8.6 concepts in class and an average of 11.1 notions in IMAGINE $\left(\mathrm{M}_{\mathrm{CL}}=8.62, \mathrm{SD}_{\mathrm{CL}}=3.77, \mathrm{M}_{\mathrm{IMG}}=11.14, \mathrm{SD}_{\mathrm{IMG}}=2.61\right)$. Results are reversed in the third tertile, in which children in the control group learned an average of 27.6 concepts, compared to 25.5 acquired by the children in the experimental group $\left(\mathrm{M}_{\mathrm{CL}}=27.66, \mathrm{SD}_{\mathrm{CL}}=4.24, \mathrm{M}_{\mathrm{IMG}}=25.5, \mathrm{SD}_{\mathrm{IMG}}=2.97\right) . \mathrm{In}$ the second tertile, children learned an average of 17 concepts, regardless the learning context $\left(\mathrm{M}_{\mathrm{CL}}=17.66, \mathrm{SD}_{\mathrm{CL}}=3.61\right.$, $\left.\mathrm{M}_{\mathrm{IMG}}=17.29, \mathrm{SD}_{\mathrm{IMG}}=1.25\right)$.

The effects of 50 days' holiday, in which students have been engaged with homework not related to this study topics, were particularly noticeable in all participants' $\mathrm{T}_{2}$ scores. Values on Memory Loss (MR), that is the number of forgotten notions between $T_{1}$ and $T_{2}$, show that students from first tertile forgot in average 7.7 and 6.6 notions in the control and experimental group respectively $\left(\mathrm{M}_{\mathrm{CL}}=7.71, \mathrm{SD}_{\mathrm{CL}}=5.21, \mathrm{M}_{\mathrm{IMG}}\right.$ $\left.=6.67, \mathrm{SD}_{\mathrm{IMG}}=3.01, \mathrm{p}<0.0001\right)$. Almost the same difference occurred for second tertile students, who lost 8.7 notions in the control group against 7.3 notions in the experimental group $\left(\mathrm{M}_{\mathrm{CL}}=8.67, \mathrm{SD}_{\mathrm{CL}}=3.7, \mathrm{M}_{\mathrm{IMG}}=7.28, \mathrm{SD}_{\mathrm{IMG}}=7.47\right.$, $\mathrm{p}<0.0001)$. The highest MR can be noticed in the third tertile, in which students who learnt in classroom forgot between 8 and 24 notions (AVG. MR: -16) and those who practised in IMAGINE forgot between none and 11 notions (AVG. MR: -5) $\left(\mathrm{M}_{\mathrm{CL}}=15.78, \mathrm{SD}_{\mathrm{CL}}=8.12, \mathrm{M}_{\mathrm{IMG}}=5, \mathrm{SD}_{\mathrm{IMG}}=6.37\right.$, $\mathrm{p}<0.0001)$.

Overall, students who have practiced in IMAGINE have shown they long-term retained many more notions than those who exercised in classroom. In particular, as depicted in Figure 6, IMAGINE students retained an average of 3 notions more than classroom students in the first tertile, 1 notion more in the second tertile, and 9 notions more in the third tertile.

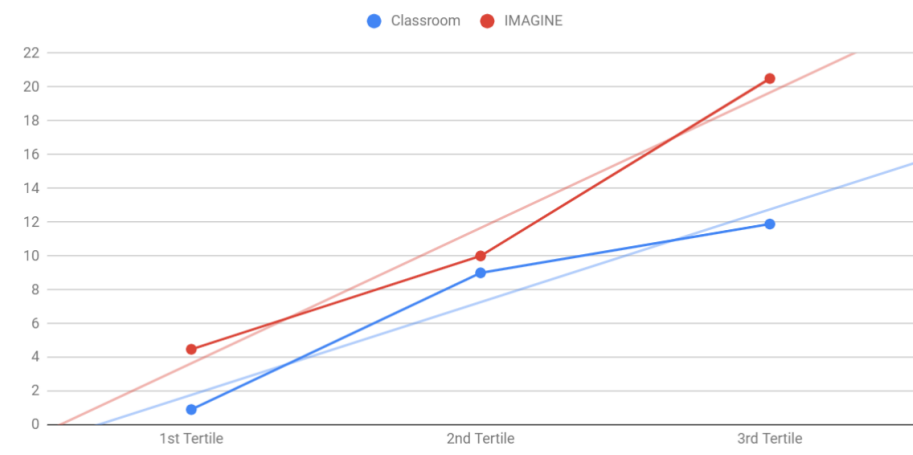

Figure 6: Classroom vs IMAGINE Long term Retention (LR)

Finally, it was found that IMAGINE has an average Content Retention Rate (CRR) of 5.8 contents/hour and an average Active Learning Turn Efficacy (ALTE) of 1.5 contents/turn, whereas the classroom has an average CRR of 3.6 contents/hour and an average ALTE of 0.9 contents/turn.

\section{DISCUSSION}

In this section we first answer the research question that led to this investigation and then discuss about possible implications and speculations of using interactive environments such as IMAGINE in daily educational activities.

\section{Research question}

Can learning benefit from an immersive embodied approach compared to a traditional paper-based classroom approach?

All 48 children retained a lot. Each child memorized an average of 18 concepts in the short term and retained 7 notions when learning in the classroom and more than 11 notions when learning in IMAGINE in the long term. Differences in Short term Retention (Figure 5) can be interpreted as follows.

Children with more learning difficulties found it easier to memorize within IMAGINE. The better outcome could be ascribed to the effect of the wide range of different interaction modes and the various senses involved, which allowed them to more easily find the learning modality suitable for their natural inclinations. In addition, the evident greater ease with which the teacher has carried out teaching sessions within IMAGINE, allows us to deduce that it acted as a catalyst of attention, thus improving retention. On the other hand, during the exercise sessions, we could notice how some of the classroom children, who then scored high, practised autonomously ahead in the execution of the exercises, waiting for the teacher and the rest of the class only in case they were not able to provide an answer to the task or when correcting it. We believe that children's autonomy in class favoured their retention rate in the short-term.

Learning depends on (especially long-term) memory as it provides the framework to which new knowledge is linked by association and inference (understanding) and, viceversa, because it lets us store and retrieve learned information $[74,20]$. Under psychological views, children's acquired notions were gathered through the senses and relayed to short-term memory (SR) where, through repetition and rehearsal, they were committed to the semantic (long-term) memory (LR). The evaluation of children's learning as their prolonged retention of notions (LR), highlighted how those who performed in IMAGINE lost, after 50 days, an average of 5 notions less than those who exercised in the classroom, allowing them to score almost double in the last test. These results are also confirmed when analyzing the Active Learning Turn Efficacy (ALTE): each child memorized almost double notions per turn when performing in IMAGINE, compared to when exercising in classroom. Such results can be ascribed to the embodied immersive approach provided by IMAGINE, that eventually enabled children to encode information more naturally, to consolidate it through embodied reflection, store and recall more easily and for a longer period. Moreover, given that, early in learning, students primarily have representations that are episodic in nature (e.g. representations of personally experienced events such as being in a place with friends) and, as learning continues and schematisation occurs, students' knowledge is more likely to be dominated by semantic memory representations [40], we can then attribute the positive ALTE and LR scores to the fact that children were facilitated in recalling notions because they were accessing to both episodic and semantic memory.

These results suggest that learning might benefit more from an IMAGINE approach than the traditional paper-based classroom process. 


\section{Insights}

A few studies of educational technology have focused on exploring the long-term impact of embodied interaction in authentic school environments. Starting from teachers' observations, this section describes the possible implications and the derived set of factors that may pave the ground of using immersive multimodal learning environments in education.

- Attractiveness. While lack of motivation in education can undermine the process of students learning, the opposite is also true [85]. Many children have shown great enthusiasm and impatience to interact with IMAGINE before the experimental sessions and, after them, by repeatedly asking to perform other activities within it. The same fervour was not reported in children belonging to the control group. Our results suggest that increased motivation shown by children in IMAGINE may have contributed to their higher performance.

- Role-changeability. Commenting video recordings, teachers pinpointed that children in IMAGINE changed their roles: those who were usually more introverted and less active were the ones who shot out joy and motivated their mates. We deduce IMAGINE was the role changer of these dynamics as also happened in other on-the-field studies [32].

- Sociability. The teacher who participated in the study defined IMAGINE a promising enabler for collective learning. It is generally thought that, knowledge is based on both intraindividual events and social community processes [86, 87]. In his theory of social learning [11], Albert Bandura points out that learning not only involves direct contact with objects, but also takes place through indirect experiences, developed through the observation of other people. This mechanism of spontaneous involvement allowed the teacher to engage in discussions in IMAGINE more easily. Given these premises, it could be interesting to further study those particular aspects of knowledge creation in working groups and the transfer methods happening between them.

- Physical Activity. IMAGINE can eventually enhance physical activity over more traditional sedentary classroom-based methods increasing students health and fitness. Some studies demonstrated that physical exercise affects brain plasticity and influences cognition and well-being by increasing cerebral blood flow, maximal oxygen consumption and delivery [92].

- Affective Activity. Supporting social constructivism intertwined with high level of interaction can support improvement in both cognitive and affective domains as shown in previous studies $[13,23,58,24]$. IMAGINE generated in children a sense of active belonging to the group, which began to rejoice in response to the correct answers given by the companions and to give advice and suggestions in moments of uncertainty. - Verisimilitude. Children not only shared a common experience, but they were also projected in a micro virtual world, representation of a real but less complex reality [26, 76]. Verisimilitude plays a critical role in children's learning, enhancing concrete thinking in naturalistic scenarios; eliciting consciousness and awareness which accompany different aspects of memory; exposing children to real learning scenarios, easier to generalize to real world situations; and, over an extended period, transforming isolated memory episodes to conceptual knowledge [17, 29, 71, 83].
- Teachers' role and sustainability. Given the results from the study, one could argue that providing the best learning environment means offering immersive and embodied experiences. This is partially true, but is worth noticing that without the continuous mediation role of the teacher, this technology would have less potential. Teachers create or organize learning material, and design the educational learning methods and strategies. Solutions like IMAGINE are just "tools". Exploiting them to provide powerful learning opportunities based on new educational methods depends on the teachers' understanding of the potential and limitations of these tools, and their ability of technology appropriation, i.e., of adopting and adapting technology to integrate it into their practices and teaching routines. This radical change will have to account veteran teachers, generally considered "digital immigrants", and novel teachers, generally considered "digital natives" and to provide methods to technologically literate the first group and to develop a pedagogy that includes interactive teaching and learning for the latter one [73]. It is thus necessary for institutions to take informed decisions and move towards a sustainable technology-driven embodied education.

- Performability. We could interpret some of the above discussed behaviors of children through the lens of performative experience design $[89,78]$. IMAGINE provides a performative context: the locus of interaction expands from the virtuality of the projections to the physical space and from a personal context to a social and public context. Like in a performance, activities in IMAGINE have "diachronic structure" [89]: a beginning, a sequence of isolable phases, and an end. During interaction, the user's body is up and on display in a performative manner in front of the class, and children are operators of, performers in, and spectators to their own - and their peers - interactions. We could therefore regard IMAGINE as a "Technology-Mediated Performative Environment" and ascribe its value for and impact on learning to the performative nature of IMAGINE experiences: the engagement, emotions, and ego investment that are associated with public performance are intrinsically more motivational compared to sitting and merely writing answers or speaking aloud.

\section{Directions for enhancing IMAGINE}

Among the several new features of IMAGINE, we report some hints about possible future enhancements in terms of user experience, system-supplied capabilities and in-depth research.

- Co-creatability. Different studies suggest that involving children as co-creators and collaborative problem solvers make learning more appraisable [22]. By allowing children to configure their environment, education is more active, engaging and student-centered and it helps students to develop higherlevel thinking ability, to increase retention and self-esteem, and to enhance their performance [8].

- Adaptivity. As demonstrated in previous works [18, 27, 38], technology is able to automatically track each learner's improvement. Digital technology, differently from analog solutions, will eventually be able to identify users' level of knowledge about the subject and their mastery with respect to the proposed motion-based interactions. This would allow the system to provide challenges that are always engaging and stimulating, allowing each student an adaptable learning experience. We believe that through adaptivity it is possible to 
achieve the objective of "bilateral inclusiveness", which not only provides children with special educational needs with the tools necessary for their education, but also stimulates those with the greatest ease of learning to achieve better results.

- Inclusivity. IMAGINE supported both short and long term retention in children with greater difficulties and empowered high performers on a prolonged time. This environment holds promise in supporting children with special educational needs (SEN) in mainstream education, given its plethora of interaction modalities and stimulus that can be carefully selected by the teacher to give SEN children the best tailored experience.

- Trackability. Profiling students and tracking their results will eventually serve not only the child itself but also teachers (who will adapt their teaching according to children's improvements), parents and the entire school community. Additional use of technologies such as bio-signal sensors, wearable body sensing equipment and EEG should be able to shed a light on the inseparable link between body and brain and therefore, inform a potential framework for embodied learning [56].

- Embodiment. For the purpose of this study, activities in IMAGINE were developed to be comparable with textbookstyle tasks; still, the system architecture, including the usage of the Kinect motion sensor, was designed with the intent of enabling richer embodied experiences that take advantages of the affordances of the media, the immersivity of the environment, and the representational power of movements and gestures. As an example of higher embodied for the Solar System learning experience, players could explore the composition of planets using connected textured tactile materials, experience crusts temperatures making use of heat lamps and refreshing fans, using distance between their hands to describe planets sizes, or use their body to mimic planets position and explore how they reciprocally move.

\section{LIMITATIONS}

Although the empirical study has been designed very carefully, there were some limitations. First, the data are self-reported, introducing several potential bias such as selective memory, telescoping, attribution, and exaggeration. The study procedure and data analysis were mostly organized before performing the study and commented before the results publication. Still we had to face, as common in any study, different unexpected circumstances that may have induced educators and researchers to hypothesize some facts afterwards (known as harking [49]).

Second, as technology limitation, we found out during the study that IMAGINE introduced some small delays that could have distorted the experience of our participants. On the other hand, the multimodality and multisensoriality of the interactive environment eventually influenced participants' focus of attention and related performances.

Third, this research was conducted on a short time range. Therefore, to generalize the results for larger groups and statistically evaluate the acquired notions, the study should be replicated for a longer period and with more participants. Within a longer time frame, running similar sessions with more participants would allow analyzing post-novelty effects, analysis and generalization of effects, and consolidation of learning.
Finally, the content, the methods, the environmental and subjective factors used to teach in IMAGINE and in classroom affected children's engagement and attention. Although we kept the same interaction methods, trying to change only the instructional treatment variable, we were not able to control all variables, such as the variability between the subjects, their learning attitude, or their preferences; in addition, the novelty effect may have played in favor of IMAGINE.

\section{CONCLUSIONS AND FUTURE WORK}

Despite nearly two decades of research on embodied learning tools, we are still a long way from knowing how best to exploit the power of embodied technologies to support learning. The individual differences among learners and the complexity of the learning process, which involves multiple intrinsic factors, contribute to make the research in this field particularly challenging and critical. Despite these and other limitations, research is making progress. The results of our empirical study are encouraging, as we detected improvements in participants' long term retention skills that could be ascribed to the experiences in IMAGINE. From a broader perspective, our research suggests that motion-based immersive technology to support embodied learning activities in regular school contexts is a promising approach, and smart spaces characterized by low-medium embodiment like IMAGINE might lead to revisit existing educational processes as well to reinvent new ones.

Overall, our work contributes to the educational technology research community by providing a deeper understanding of how we use embodied learning technologies in real learning contexts and outlines some hints of how these technologies could be improved in the future.

In the future we plan to leverage the potential of motion sensing technologies by designing full-body activities that make use of iconic and representational gestures so to take full advantage of the affordances of IMAGINE and we will expand the study to a larger number of participants who will be involved for a much longer time, tracking their performances within each learning session. Such research would also support the creation of a corpus of data on children's bodily learning, unique in this field and valuable for educational practice and research.

\section{ACKNOWLEDGEMENTS}

We gratefully thank managers, teachers and students at Via Sturzo school in Cornaredo (Italy). We thank students and researchers behind the design, development and evaluation of IMAGINE. Above all, we wish to acknowledge: Giulia Cosentino, Mattia Gianotti, Micol Spitale, Lucia di Terlizzi, Barbara Bonadies.

Note: Images, exercise and test sheets, and study materials are available in the supplementary materials. 


\section{REFERENCES}

[1] Dor Abrahamson and Robb Lindgren. 2014. Embodiment and embodied design. The Cambridge handbook of the learning sciences 2 (2014), 358-376.

[2] Takayuki Adachi, Masafumi Goseki, Keita Muratsu, Hiroshi Mizoguchi, Miki Namatame, Masanori Sugimoto, Fusako Kusunoki, Etsuji Yamaguchi, Shigenori Inagaki, and Yoshiaki Takeda. 2013. Human SUGOROKU: full-body interaction system for students to learn vegetation succession. In Proceedings of the 12th International Conference on Interaction Design and Children. ACM, 364-367.

[3] Giovanni Agosta, Luca Borghese, Carlo Brandolese, Francesco Clasadonte, William Fornaciari, Franca Garzotto, Mirko Gelsomini, Matteo Grotto, Cristina Frà, Danny Noferi, and others. 2015. Playful Supervised Smart Spaces (P3S)-A Framework for Designing, Implementing and Deploying Multisensory Play Experiences for Children with Special Needs. In 2015 Euromicro Conference on Digital System Design. IEEE, 158-164.

[4] Shirley Agostinho, Sharon Tindall-Ford, Paul Ginns, Steven J Howard, Wayne Leahy, and Fred Paas. 2015. Giving learning a helping hand: finger tracing of temperature graphs on an iPad. Educational Psychology Review 27, 3 (2015), 427-443.

[5] Michael W Alban and Colleen M Kelley. 2013. Embodiment meets metamemory: Weight as a cue for metacognitive judgments. Journal of Experimental Psychology: Learning, Memory, and Cognition 39, 5 (2013), 1628.

[6] Bashar Altakrouri and Andreas Schrader. 2012. Towards dynamic natural interaction ensembles. In Fourth International Workshop on Physicality. 1.

[7] Stamatina Anastopoulou, Mike Sharples, and Chris Baber. 2011. An evaluation of multimodal interactions with technology while learning science concepts. British Journal of Educational Technology 42, 2 (2011), 266-290.

[8] Oso Senny Oluwatumbi and. 2017. Innovative Pedagogies: Panacea for Students Academic Performance. International Journal of Trend in Scientific Research and Development Volume-1, Issue-6 (Oct. 2017), 1013-1019. DOI :

http://dx.doi.org/10.31142/ijtsrd5759

[9] Janice L Anderson and Steven D Wall. 2016. Kinecting physics: Conceptualization of motion through visualization and embodiment. Journal of Science Education and Technology 25, 2 (2016), 161-173.

[10] Saskia Bakker, Elise Van Den Hoven, and Alissa N Antle. 2011. MoSo tangibles: evaluating embodied learning. In Proceedings of the fifth international conference on Tangible, embedded, and embodied interaction. ACM, 85-92.
[11] Albert Bandura and Richard H Walters. 1977. Social learning theory. Vol. 1. Prentice-hall Englewood Cliffs, NJ.

[12] David Birchfield, Thomas Ciufo, and Gary Minyard. 2006. SMALLab: a mediated platform for education. In ACM SIGGRAPH 2006 Educators program. ACM, 33.

[13] Phyllis Blumenfeld, Barry J Fishman, Joseph Krajcik, Ronald W Marx, and Elliot Soloway. 2000. Creating usable innovations in systemic reform: Scaling up technology-embedded project-based science in urban schools. Educational psychologist 35, 3 (2000), 149-164.

[14] Doug A Bowman and Ryan P McMahan. 2007. Virtual reality: how much immersion is enough? Computer 40, 7 (2007), 36-43.

[15] Neon Brooks and Susan Goldin-Meadow. 2016. Moving to learn: How guiding the hands can set the stage for learning. Cognitive Science 40, 7 (2016), 1831-1849.

[16] Anna Carreras and Narcís Parés. 2009. Designing an interactive installation for children to experience abstract concepts. In New trends on human-computer interaction. Springer, 33-42.

[17] Martin A Conway, John M Gardiner, Timothy J Perfect, Stephen J Anderson, and Gillian M Cohen. 1997. Changes in memory awareness during learning: The acquisition of knowledge by psychology undergraduates. Journal of Experimental Psychology: General 126, 4 (1997), 393.

[18] Giulia Cosentino, Giulia Leonardi, Mirko Gelsomini, Micol Spitale, Mattia Gianotti, Franca Garzotto, and Venanzio Arquilla. 2019. GENIEL: an auto-generative intelligent interface to empower learning in a multi-sensory environment.. In IUI Companion. 27-28.

[19] Tanja Dackermann, Ursula Fischer, Hans-Christoph Nuerk, Ulrike Cress, and Korbinian Moeller. 2017. Applying embodied cognition: from useful interventions and their theoretical underpinnings to practical applications. Zdm 49, 4 (2017), 545-557.

[20] Milton J Dehn. 2011. Working memory and academic learning: Assessment and intervention. John Wiley \& Sons.

[21] D DeSutter and M Stieff. 2017. Teaching students to think spatially through embodied actions: Design principles for learning environments in science, technology, engineering, and mathematics. Cognitive research: principles and implications 2, 1 (2017), 22.

[22] John Dewey. 1986. Experience and education. In The Educational Forum, Vol. 50. Taylor \& Francis, 241-252.

[23] Yehudit Judy Dori and Miri Barak. 2001. Virtual and physical molecular modeling: Fostering model perception and spatial understanding. Journal of Educational Technology \& Society 4, 1 (2001), 61-74. 
[24] Yehudit Judy Dori, Miri Barak, and Noam Adir. 2003. A Web-based chemistry course as a means to foster freshmen learning. Journal of Chemical Education 80, 9 (2003), 1084.

[25] Adam K Dubé and Rhonda N McEwen. 2015. Do gestures matter? The implications of using touchscreen devices in mathematics instruction. Learning and Instruction 40 (2015), 89-98.

[26] Carolien Duijzer, Marja Van den Heuvel-Panhuizen, Michiel Veldhuis, Michiel Doorman, and Paul Leseman. 2019. Embodied Learning Environments for Graphing Motion: a Systematic Literature Review. Educational Psychology Review (2019), 1-33.

[27] Tanya Elias. 2011. Learning analytics. Learning (2011), $1-22$.

[28] Celestin Freinet. 1993. Education through work: A model for child-centered learning. Vol. 19. Edwin Mellen Press.

[29] John M Gardiner and Rosalind I Java. 1993. Recognising and remembering. (1993).

[30] Franca Garzotto and Mirko Gelsomini. 2018. Magic Room: A Smart Space for Children with Neurodevelopmental Disorder. IEEE Pervasive Computing 17, 1 (2018), 38-48.

[31] Mirko Gelsomini, Giulia Cosentino, Micol Spitale, Mattia Gianotti, Davide Fisicaro, Giulia Leonardi, Fabiano Riccardi, Agnese Piselli, Eleonora Beccaluva, Barbara Bonadies, and others. 2019. Magika, a Multisensory Environment for Play, Education and Inclusion. In Extended Abstracts of the 2019 CHI Conference on Human Factors in Computing Systems. ACM, LBW0277.

[32] Mirko Gelsomini, Annalisa Rotondaro, Giulia Cosentino, Mattia Gianotti, Fabiano Riccardi, and Franca Garzotto. 2018. On the Effects of a Nomadic Multisensory Solution for Children's Playful Learning. In Proceedings of the 2018 ACM International Conference on Interactive Surfaces and Spaces. ACM, 189-201.

[33] Paul Ginns, Fang-Tzu Hu, Erin Byrne, and Janette Bobis. 2016. Learning by tracing worked examples. Applied Cognitive Psychology 30, 2 (2016), 160-169.

[34] Arthur M Glenberg. 2017. How reading comprehension is embodied and why that matters. International Electronic Journal of Elementary Education 4, 1 (2017), 5-18.

[35] Arthur M Glenberg, Tiana Gutierrez, Joel R Levin, Sandra Japuntich, and Michael P Kaschak. 2004. Activity and imagined activity can enhance young children's reading comprehension. Journal of educational psychology 96, 3 (2004), 424.

[36] Kaj Grønbæk, Ole Sejer Iversen, Karen Johanne Kortbek, Kaspar Rosengreen Nielsen, and Louise
Aagaard. 2007. Interactive floor support for kinesthetic interaction in children learning environments. In IFIP Conference on Human-Computer Interaction. Springer, 361-375.

[37] Insook Han and John B Black. 2011. Incorporating haptic feedback in simulation for learning physics. Computers \& Education 57, 4 (2011), 2281-2290.

[38] Margo Hanna. 2004. Data mining in the e-learning domain. Campus-wide information systems 21, 1 (2004), 29-34.

[39] Anja Hashagen, Corinne Büching, and Heidi Schelhowe. 2009. Learning abstract concepts through bodily engagement: a comparative, qualitative study. In Proceedings of the 8th International Conference on Interaction Design and Children. ACM, 234-237.

[40] D Herbert. 1999. What do students remember from lectures? The role of episodic memory on early learning. In combined Australian Association for Research in Education - New Zealand Association for Research in Education Conference, Melbourne.

[41] Simon Holland, Paul Marshall, Jon Bird, Sheep Dalton, Richard Morris, Nadia Pantidi, Yvonne Rogers, and Andy Clark. 2009. Running up Blueberry Hill: Prototyping whole body interaction in harmony space. In Proceedings of the 3rd international Conference on Tangible and Embedded interaction. ACM, 93-98.

[42] Fang-Tzu Hu, Paul Ginns, and Janette Bobis. 2015. Getting the point: Tracing worked examples enhances learning. Learning and Instruction 35 (2015), 85-93.

[43] Samuel Aldo Iacolina, Alessandro Lai, Alessandro Soro, and Riccardo Scateni. 2010. Natural Interaction and Computer Graphics Applications.. In Eurographics italian chapter conference. 141-146.

[44] Vanderbilt University Iris Center for Faculty Enhancement. 2019. Star Legacy Modules. https://iris.peabody.vanderbilt. edu/module/hpl/ cresource/q2/p07. (2019).

[45] Mina C Johnson-Glenberg, David Birchfield, Philippos Savvides, and Colleen Megowan-Romanowicz. 2011. Semi-virtual embodied learning-real world stem assessment. In Serious Educational Game Assessment. Brill Sense, 241-257.

[46] Mina C Johnson-Glenberg, David A Birchfield, Lisa Tolentino, and Tatyana Koziupa. 2014. Collaborative embodied learning in mixed reality motion-capture environments: Two science studies. Journal of Educational Psychology 106, 1 (2014), 86.

[47] Mina C Johnson-Glenberg, Tatyana Koziupa, David Birchfield, and Kyle Li. 2011. Games for learning in embodied mixed-reality environments: Principles and results. In Proceedings of the 7th international conference on Games+ Learning + Society Conference. ETC Press, 129-137. 
[48] Mina C Johnson-Glenberg, Colleen Megowan-Romanowicz, David A Birchfield, and Caroline Savio-Ramos. 2016. Effects of embodied learning and digital platform on the retention of physics content: Centripetal force. Frontiers in psychology 7 (2016), 1819.

[49] Norbert L Kerr. 1998. HARKing: Hypothesizing after the results are known. Personality and Social Psychology Review 2, 3 (1998), 196-217.

[50] Wolfgang Klimesch. 2013. The structure of long-term memory: A connectivity model of semantic processing. Psychology Press.

[51] Alice Y Kolb and David A Kolb. 2005. Learning styles and learning spaces: Enhancing experiential learning in higher education. Academy of management learning \& education 4, 2 (2005), 193-212.

[52] David A Kolb. 2007. The Kolb learning style inventory. Hay Resources Direct Boston, MA.

[53] David A Kolb. 2014. Experiential learning: Experience as the source of learning and development. FT press.

[54] David A Kolb, Richard E Boyatzis, Charalampos Mainemelis, and others. 2001. Experiential learning theory: Previous research and new directions.

Perspectives on thinking, learning, and cognitive styles 1, 8 (2001), 227-247.

[55] Carly Kontra, Daniel J Lyons, Susan M Fischer, and Sian L Beilock. 2015. Physical experience enhances science learning. Psychological science 26, 6 (2015), 737-749.

[56] Panagiotis Kosmas, Andri Ioannou, and Symeon Retalis. 2018. Moving bodies to moving minds: A study of the use of motion-based games in special education. TechTrends 62, 6 (2018), 594-601.

[57] Panagiotis Kosmas, Andri Ioannou, and Panayiotis Zaphiris. 2019. Implementing embodied learning in the classroom: effects on children's memory and language skills. Educational Media International 56, 1 (2019), 59-74.

[58] Joseph Krajcik, Ron Marx, Phyllis Blumenfeld, Elliot Soloway, and Barry Fishman. 2000. Inquiry Based Science Supported by Technology: Achievement among Urban Middle School Students. (2000).

[59] George Lakoff and Rafael E Núñez. 2000. Where mathematics comes from: How the embodied mind brings mathematics into being. AMC 10, 12 (2000), 720-733.

[60] Wan-Ju Lee, Chi-Wen Huang, Chia-Jung Wu, Shing-Tsaan Huang, and Gwo-Dong Chen. 2012. The effects of using embodied interactions to improve learning performance. In 2012 IEEE 12th International Conference on Advanced Learning Technologies. IEEE, 557-559.
[61] Mei Yii Lim, Karin Leichtenstern, Michael Kriegel, Sibylle Enz, Ruth Aylett, Natalie Vannini, Lynne Hall, and Paola Rizzo. 2011. Technology-enhanced role-play for social and emotional learning context-Intercultural empathy. Entertainment Computing 2, 4 (2011), 223-231.

[62] Robb Lindgren and Mina Johnson-Glenberg. 2013. Emboldened by embodiment: Six precepts for research on embodied learning and mixed reality. Educational Researcher 42, 8 (2013), 445-452.

[63] Laura Malinverni and Narcis Pares. 2014. Learning of abstract concepts through full-body interaction: a systematic. Educational Technology \& Society 17, 4 (2014), 100-116.

[64] Myrto-Foteini Mavilidi, Anthony D Okely, Paul Chandler, Dylan P Cliff, and Fred Paas. 2015. Effects of integrated physical exercises and gestures on preschool children's foreign language vocabulary learning. Educational Psychology Review 27, 3 (2015), 413-426.

[65] Edward F Melcer and Katherine Isbister. 2016. Bridging the physical divide: a design framework for embodied learning games and simulations. In Proceedings of the 2016 CHI Conference Extended Abstracts on Human Factors in Computing Systems. ACM, 2225-2233.

[66] RE Nunez and WJ Freeman. 1999. Restoring to cognition the forgotten primacy of action, intention and emotion. Journal of Consciousness Studies 6, 11-12 (1999), ix-Xx.

[67] Narcís Parés, Anna Carreras, and Jaume Durany. 2005. Generating meaning through interaction in a refreshing interactive water installation for children. In Proceedings of Interaction Design and Children. ACM New York, NY, 218-223.

[68] Jean Piaget. 1964. Part I: Cognitive development in children: Piaget development and learning. Journal of research in science teaching 2, 3 (1964), 176-186.

[69] Friedemann Pulvermüller and Luciano Fadiga. 2010. Active perception: sensorimotor circuits as a cortical basis for language. Nature reviews neuroscience 11, 5 (2010), 351.

[70] Luis Radford, Caroline Bardini, Cristina Sabena, Pounthioun Diallo, and Athanase Simbagoye. 2005. On Embodiment, Artifacts, and Signs: A Semiotic-Cultural Perspective on Mathematical Thinking. International Group for the Psychology of Mathematics Education 4 (2005), 113-120.

[71] Suparna Rajaram and Henry L Roediger III. 1997. Remembering and knowing as states of consciousness during retrieval. Scientific approaches to consciousness 11 (1997), 213-40.

[72] Margina Ruiter, Sofie Loyens, and Fred Paas. 2015. Watch your step children! Learning two-digit numbers through mirror-based observation of self-initiated body movements. Educational Psychology Review 27, 3 (2015), 457-474. 
[73] Diallo Sessoms. 2008. Interactive instruction: Creating interactive learning environments through tomorrow's teachers. International Journal of Technology in Teaching and Learning 4, 2 (2008), 86-96.

[74] Lauralee Sherwood. 2015. Human physiology: from cells to systems. Cengage learning.

[75] Alexander Skulmowski and Günter Daniel Rey. 2018. Embodied learning: introducing a taxonomy based on bodily engagement and task integration. Cognitive research: principles and implications 3, 1 (2018), 6.

[76] Joan Solomon, Richard Bevan, A Frost, H Reynolds, M Summers, and C Zimmerman. 1991. Can pupils learn through their own movements? A study of the use of a motion sensor interface. Physics Education 26, 6 (1991), 345.

[77] Norman E Spear. 2014. The Processing of Memories (PLE: Memory): Forgetting and Retention. Psychology Press.

[78] Jocelyn Spence. 2016. Performative experience design. Springer.

[79] Marilee Sprenger. 1999. Learning and memory: The brain in action. ASCD.

[80] Erik F Strommen. 1993. Is it easier to hop or walk? development issues in interface design.

Human-Computer Interaction 8, 4 (1993), 337-352.

[81] Cathy Tran, Brandon Smith, and Martin Buschkuehl. 2017. Support of mathematical thinking through embodied cognition: Nondigital and digital approaches. Cognitive Research: Principles and Implications 2, 1 (2017), 16

[82] Endel Tulving. 1985a. Elements of episodic memory. (1985).

[83] Endel Tulving. 1985b. Memory and consciousness. Canadian Psychology/Psychologie canadienne 26, 1 (1985), 1 .
[84] Mark E Turner. 1999. Child-Centered Learning and Music Programs: Child-centered learning in music programs for young children can foster their emotional, social, cognitive, and musical development. Music Educators Journal 86, 1 (1999), 30-51.

[85] Eskja Vero and Edi Puka. 2017. The importance of motivation in an educational environment. Formazione \& Insegnamento XV 15, 1 (2017), 57-66.

[86] Sandrine Vieillevoye and Nathalie Nader-Grosbois. 2008. Self-regulation during pretend play in children with intellectual disability and in normally developing children. Research in developmental disabilities 29, 3 (2008), 256-272.

[87] James V Wertsch. 1985. Vygotsky and the social formation of mind. Harvard University Press.

[88] Mark Wexler, Stephen M Kosslyn, and Alain Berthoz. 1998. Motor processes in mental rotation. Cognition 68, 1 (1998), 77-94.

[89] Julie R Williamson and Lone Koefoed Hansen. 2012. Designing performative interactions in public spaces. In Proceedings of the Designing Interactive Systems Conference. ACM, 791-792.

[90] Margaret Wilson. 2002. Six views of embodied cognition. Psychonomic bulletin \& review 9, 4 (2002), 625-636.

[91] Robert A Wilson and Lucia Foglia. 2011. Embodied cognition. (2011).

[92] Ania Zubala, Stephen MacGillivray, Helen Frost, Thilo Kroll, Dawn A Skelton, Anna Gavine, Nicola M Gray, Madalina Toma, and Jacqui Morris. 2017. Promotion of physical activity interventions for community dwelling older adults: a systematic review of reviews. PloS one 12, 7 (2017), e0180902. 